\title{
Effects of Zolpidem on Local Cerebral Glucose Metabolism during Non-REM Sleep in Normal Volunteers: A Positron Emission Tomography Study
}

\author{
J. Christian Gillin, M.D., Monte S. Buchsbaum, M.D., Dirceu C. Valladares-Neto, M.D., \\ Charles Chong-Hiwa Hong, M.D., Ph.D., Erin Hazlett, Ph.D., Salomon Z. Langer, M.D., \\ and Joseph Wu, M.D.
}

Using positron emission tomography a'ith

fluorodeoxyglucose ( ${ }^{8} \mathrm{FDG}$ or FDG), we compared the effects of zolpidem (10 $\mathrm{mg}$ ), an imidazopyridine hypnotic, which is relatively selective for the $B Z_{1}$ or omega receptor and placebo on cerebral glucose metabolism during the first non-REM sleep period of 12 young nomal zolunteers. Plasma zolpidem planmacokinetics iaried considerably among subjects, and plasma concentrations arere lower than usually reported. In general, the effects of zolpidem on local cerebral glucose metabolism anried directly with plasma concentrations of zolpidem. Zolpidem induced changes in local cerebral glucose metabolism were unevenly distributed throughout the brain and were greater in subcortical areas than lateral cortical areas. Significant negative correlations were found between change in local absolute glucose metabolic rate (calculated by subtracting individual data on placebo nights from that on zolpidem nights) and plasma concentration of zolpidem for the following areas: medial frontal cortex, cingulate gyrus, putamen, thalamus, and hippocampus. The effects of zolpidem on local cerebral glucose metabolism were partially but not closely related to the reported density of $B Z_{1}$ receptors.

[Neuropsychopharmacology 15:302-313, 1996]
KEY WORDS: Benzodiazepine receptor subtype; Omega receptor; Insomnia; Imidazopyridine; Cerebral metabolism; FDG; PET

From the Department of Psychiatry (JCG, CC-HH), Lniversity of California, San Diego, and San Diego VA Medical Center; the Department of Psychiatry (MSB; EH), Mt. Sinai School of Medicine, New York; the Department of Psychiatry (DCV-N), Fundação Hospitalar do Estado de Minas Gerais, Brazil; the Synthelabo Recherche (LERS) (SL), Paris, France; and the Department of Psychiatry (MB, $\mathrm{EH}, \mathrm{JW})$, University of California, Irvine.

Address correspondence to: J. Christian Gillin, M.D., University of California, San Diego, Psychiatry Service (116A), San Diego Veterans Administration Medical Center, $3350 \mathrm{La}$ Jolla Village Drive, La Jolla, CA 92161

Received January 13, 1995; revised August 7, 1995; accepted October 17,1995 .
Zolpidem is a relatively new imidazopyridine sleeping pill. Hypnotic effects have been demonstrated in normal volunteers (Scharf 1991; Fairweather et al. 1992), acute and chronic insomniacs (Jonas et al. 1992; Shaw et al. 1992), the elderly (Roger et al. 1993), and with chronic treatment (Maarek et al. 1992). Unlike the currently prescribed benzodiazepine hypnotics in the United States (flurazepam, triazolam, temazepam, quazepam, estazolam), zolpidem apparently has little effect on EEG sleep patterns, such as sleep stages 3 and 4 (delta), which are typically decreased by the traditional benzodiazepines. In addition, even though it has a short half-life of approximately 2 to 3 hours, similar to that of triazolam, zolpidem has been infrequently associated with some of the side effects reported with triazolam or other 
short-half-life benzodiazepines, such as rebound insomnia or amnesia. Likewise, zolpidem apparently has few anxiolytic, anticonvulsant, or muscle relaxant effects, which are also characteristic properties of the benzodiazepines.

The pharmacological differences between zolpidem and the benzodiazepines have been attributed to the relative selectivity of receptor subtype binding of zolpidem compared with the benzodiazepines. Although it differs in chemical structure from the benzodiazepines, zolpidem does bind mainly to the $\mathrm{BZ}_{1}$ or omega benzodiazepine receptor subtype, one of three benzodiazepine receptor subtypes. In contrast to the benzodiazepine hypnotics, zolpidem does not bind tightly to the other two benzodiazepine receptor subtypes, omega and omega 3 (Langer et al. 1988). Modulation of the $\mathrm{GABA}_{\mathrm{A}}$ receptor chloride channel macromolecular complex by the alpha subunit apparently determines the three different receptor subtypes (Langer et al. 1988; Benavides et al. 1993a, 1993b; Marksitzer et al. 1993; Mertens et al. 1993; Wafford et al. 1993). The $B Z_{1}$ receptor presumably conveys the sedative effects of these two classes of hypnotics, whereas the anxiolytic, anticonvulsant, and muscle-relaxant properties are mediated by the other receptor subtypes. The omega ${ }_{1}$ receptors have been localized primarily in layer IV of the sensorimotor cortex, cerebellum, substantia nigra, olfactory bulb, and inferior colliculus (Benavides et al. 1993b). In addition, however, binding of $\left[{ }^{3} \mathrm{H}\right]$-zolpidem to omega receptors is enhanced by GABA irrespective of the relative proportion of omega, receptors in various sites, including the lateral geniculate, olfactory tubercle, red nucleus, cingulate, and frontal-parietal cortices (Ruano et al. 1993).

In this study, we compared the effects of zolpidem and placebo on cerebral glucose metabolism rate (CGMR) during non-REM sleep in normal volunteers. To our knowledge it is the first study of the effect of any drug on cerebral glucose metabolism during sleep in humans. This study is one of a series of studies we have conducted on the relationships between CGMR and normal sleep-wake patterns in monkeys (Kennedv et al. 1982; Nakamura et al. 1983), normal rolunteers (Buchsbaum et al. 1989), and depressed patients (Ho, Gillin, Buchsbaum, Wu, and Bunney, unpublished data), as well as the effect of sleep deprivation on CGMR in normal volunteers (Wu et al. 1991a) and depressed patients (Wu et al. 1992). We and others have previously reported that CGMR, cerebral blood flow or oxygen metabolism is significantly reduced, by about $25 \%$ to $35 \%$, during non-REM sleep compared with wakefulness in monkeys (Kennedy et al. 1982; Nakamura et al. 1983), rat (Ramm and Frost 1983) and cat (Ramm and Frost 1986), and normal humans (Buchsbaum et al. 1989; Madsen et al. 1991a, 1991b; Franzini 1992; Maquet et al. 1992).
In one previous study in rats, Piercey et al. (1991) compared the effects of zolpidem and triazolam on 2-deoxyglucose metabolism and concluded that both drugs depressed brain metabolism in the same areas of the brain, that the effects of zolpidem were not mediated solely by $\mathrm{BZ}_{1}$ receptors, and that areas rich in $\mathrm{BZ}_{2}$ receptors may be more functionally significant than areas rich in $B Z_{1}$ receptors. The generalizability of this study to the effects of hypnotic drugs on human brain metabolism during sleep remains unknown, especially because the doses of zolpidem and triazolam were high (zolpidem, $10-30 \mathrm{mg} / \mathrm{kg}$; triazolam, $0.3-1 \mathrm{mg} / \mathrm{kg}$ ) and difficult to extrapolate to those used in humans as sleeping pills.

In this study, we predicted that zolpidem would have little or no effect compared with placebo on whole-brain CGMR during the first period of non-REM sleep. We did predict that zolpidem would reduce local glucose metabolism in areas relatively rich in omega receptors or where it has been reported that GABA potentiates the binding of zolpidem.

\section{METHODS}

Twelve male normal controls (mean age, 22.5, $\mathrm{SD}=1.6$, 11 white, 1 Hispanic) were studied by positron emission tomography (PET) and polysomnography during the first non-REM sleep period of the night following double-blind administration, in random order, of either zolpidem $(10 \mathrm{mg})$ or placebo capsules approximately 20 minutes before bedtime. Subjects had been previously evaluated by complete medical and psychiatric history, physical and laboratory examinations, and a baseline sleep study for adaptation to the sleep laboratory and for screening for sleep apnea and nocturnal myoclonus. None of the normal controls had a history of sleep disorders or serious medical, neurological, or psychiatric problems, alcohol or substance abuse, recent shiftwork or unusual sleep-wake schedules, or use of sleeping pills or other psychoactive medications.

Subjects received placebo on one night and zolpidem (10 $\mathrm{mg}$ PO) on another night in a double-blind, random-order design. At least 6 days separated the two study nights. Zolpidem tartrate or placebo powder was administered in identical-appearing gelatin capsules, which were prepared by Lorex Pharmaceuticals (Skokie, IL) especially for this study. Subjects maintained their normal sleep-wake patterns and retired shortly after approximately 2300 .

\section{Polygraphic Sleep Recordings}

Subjects were adapted to sleeping with polygraphic recording of EEG, EOG, submental EMG, and EKG and 
with an intravenous catheter for one night before the actual two study nights, as described in our previous study of CGMR during sleep in normal volunteers (Buchsbaum et al. 1989). Polygraphic recordings were obtained and visually scored according to the criteria of Rechtschaffen and Kales (1971), as previously described (Buchsbaum et al. 1989).

EEG was also recorded on electronic tape for later period and amplitude analysis (Pass Plus, Delta Software, San Francisco, CA). The EEG data were digitalized at $200 \mathrm{~Hz}$, and the following frequency bands were analyzed: $0.3-1.0,1-2,2-3,3-4,4-8,8-12$, and $12-$ $20 \mathrm{~Hz}$.

\section{PET Scanning}

Before PET scanning, an individually molded, thermostatic plastic head holder was made for each subject, to minimize head movement and to ensure accurate and reproducible positioning on the second night compared with the first night. Repeated studies with magnetic resonance imaging (MRI) have indicated an average repositioning error of about $2 \mathrm{~mm}$ (Buchsbaum et al. 1992a). Test-retest correlations for FDG PET show that it is a highly reliable method (Bartlett et al. 1991). For the PET procedure, subjects slept alone in a dark, quiet individual bedroom and were studied according to previously described methods (Buchsbaum et al. 1989). Intravenous lines of $0.9 \%$ saline drip were inserted into both arms; a 3-m polyethylene tube extended out of the room so that FDG could be administered in one arm and blood could be withdrawn from the other arm without disturbing the subject. The arm used for blood withdrawal was wrapped in a hot pack for arterialization of venous blood and was used to withdraw blood samples $(2 \mathrm{ml})$ to determine the kinetics of both FDG and zolpidem. Polygraphic tracings of EEG, EOG, EMG, and EKG were continuously monitored by visual observation and were recorded on electronic tape for later computerized measurement of period and amplitude. Approximately 20 to 25 minutes after the onset of stage 2 sleep, FDG ( $4-5 \mathrm{mCi})$ was administered intravenously over 30 seconds in one arm, while blood samples were withdrawn from the other arm. After approximately 32 minutes of FDG uptake, the subject was awakened and transferred to the adjacent scanning room. Nine planes (CTI NeuroECAT) at $10-\mathrm{mm}$ increments and parallel to the canthomeatal line (CM) were done between 45 and 100 minutes after FDG injection.

Scans were performed with both septa and shadow shields in a configuration with measured in-plane resolution of $7.6 \mathrm{~mm}$ and $10.9 \mathrm{~mm}$ resolution in the $z$-dimension (axial). A calculated attenuation correction and smooth filter were used. The scanner was calibrated each scan day, with a cylindrical phantom and compared with well-counter data.

\section{Scan Slice Selection and Processing}

Scans were transformed to glucose metabolic rate as described elsewhere (Buchsbaum et al. 1989). Regional glucose use was expressed in two ways, as absolute CGMR in mmol $/ 100 \mathrm{~g} /$ minute (in 11 subjects for technical reasons), and as relative CGMR (in 12 subjects) (ratio of regional CGMR to whole-brain mean CGMR for lateral cortex and to whole-slice mean CGMR for subcortical regions of interest). Lateral cortical regions of interest were measured using our "peel" technique (Buchsbaum et al. 1984). Ratios are reported relative to whole brain and slice to match earlier publications and to maintain a uniform comparison in other studies in which full brain metabolic rate is not collected. The correlation between ratios calculated both ways is in the 0.95 to 0.97 range. The slices were outlined with a boundary-finding algorithm, and a 2-cm-wide ring of cortex was identified and divided into lobe and gyral regions as described in Buchsbaum et al. (1989). This procedure yielded four cortical gyri or segments in each of the four lobes (frontal, parietal, temporal, and occipital) in each hemisphere for a total of 32 cortical areas. This proportional circumferential method has been widely used in functional brain imaging research (see review by Harris et al. 1991) and avoids the problems of obtaining areas of interest that lie outside the brain inherent in methods that rely on linear distances projected from the midline measured in millimeters. Subcortical and medial cortical areas were measured using the same atlas and stereotaxic method as described in Buchsbaum et al. (1989). Slices were registered across conditions using the automatically located edge. The accuracy of the current stereotaxic method against group MRI data is reported elsewhere (Buchsbaum et al. 1992a).

\section{Zolpidem Plasma Levels}

Blood samples for zolpidem were collected just before administration of capsules and afterwards at 5, 15, 30, $45,60,75$, and 90 minutes. They were spun for plasma at the end of each recording session and were frozen until assayed, approximately 3 to 4 months following the last PET scan study. The assay was done by highperformance liquid chromatography (HPLC) with fluorescence detection (Guinebault et al. 1986). The relationship between peak height ratio and concentration of zolpidem was linear in the curve ranges from 1.0 to 440 $\mathrm{ng} / \mathrm{ml}(r>999)$. The variability of the back-calculated mean concentrations of the calibration standards ranged from $0.4 \%$ to $3.7 \%$ for zolpidem. The between-day vari- 
ability did not exceed $2 \%$. The limit of detection was established to be $1 \mathrm{ng} / \mathrm{ml}$.

\section{Statistics}

CGMR data for cerebral cortex were analyzed first by four-way multivariate analysis of variance (MANOVA) in a drug- (placebo versus zolpidem) by-lobe- (frontal, parietal, temporal, occipital) by-hemisphere- (right, left) by-plasma-level-group (above and below median) design. For the medial cortical and subcortical structures, we correlated plasma concentration of zolpidem in each subject with change in CGMR (zolpidem minus placebo nights) for all brain areas.

\section{RESULTS}

\section{Plasma Zolpidem Concentrations}

The plasma concentration for zolpidem (mean $\pm S D$ ) is shown in Figure 1. The individual variation was high, reflecting great individual differences in rates of absorption. The peak plasma concentrations of zolpidem were generally low, ranging from zero to $192 \mathrm{ng} / \mathrm{ml}$. The highest concentration for each subject averaged $105 \pm$ $106 \mathrm{ng} / \mathrm{ml}$ (mean \pm SD). Plasma zolpidem levels for each subject were also averaged during the 30 minutes following the administration of FDG; the mean $\pm \mathrm{SD}$ for the 12 subjects was $56 \pm 47 \mathrm{ng} / \mathrm{ml}$.

FDG was administered intravenously to subjects $64 \pm$ 19 minutes (mean \pm SD) after the oral administration of zolpidem. When we examined the relationship between

\section{ZOLPIDEM CONCENTRATIONS}

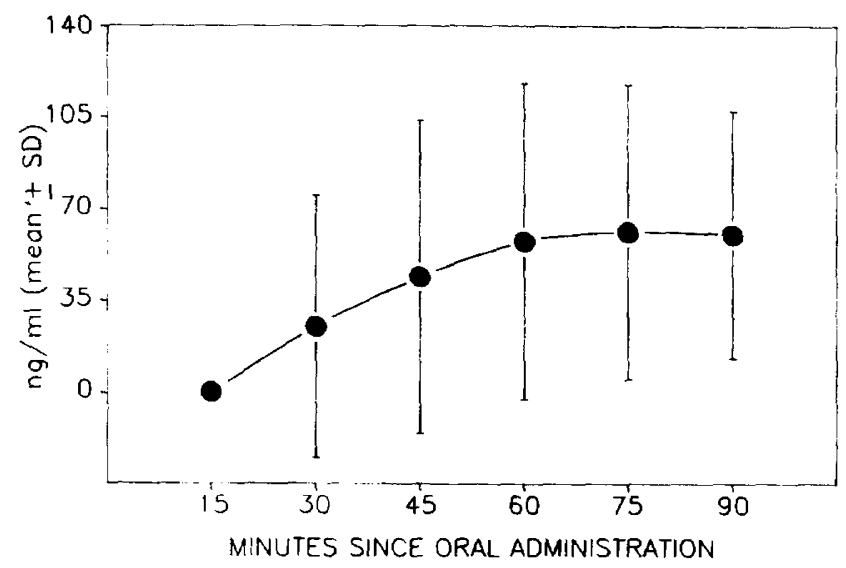

Figure 1. Plasma zolpidem concentrations (mean \pm SD) after administration of zolpidem 10 capsule by mouth. Zolpidem or placebo were administered about 20 minutes before lights-out time (about 2300 to 2315). FDG was administered about 20 to 25 minutes after onset of Stage 2 sleep, $64 \pm$ 19 minutes after oral administration of zolpidem. plasma concentrations of zolpidem and changes in local cerebral metabolism induced by zolpidem compared with placebo, the results were essentially the same whether we used the individual subject's mean plasma zolpidem level during either the 90 minutes after oral administration of zolpidem or the 30 minutes of FDG uptake. Therefore, the results presented in this article used the mean of all zolpidem values administration. We determined the effect of plasma zolpidem concentrations in two ways: (1) by dividing the subjects into low-plasma zolpidem and high-plasma zolpidem groups, based on a median split; and (2) by Pearson productmoment correlations between drug plasma levels and change in metabolic rate (zolpidem night minus placebo night) for each subject.

\section{Polygraphic Sleep Recordings}

As shown in Table 1, neither visually-scored sleep measures nor computer-scored EEG baseline crossings differed significantly between placebo and zolpidem nights. The change in sleep latency or in the number of EEG baseline crossings within each frequency bin per epoch (zolpidem minus placebo nights) did not correlate significantly with the zolpidem plasma levels either. For technical reasons, the amplitude of EEG could not be analyzed by computer-assisted methods.

\section{Absolute Glucose Metabolic Rate and Plasma Zolpidem Concentrations}

Figure 2 shows a typical scan of cerebral glucose metabolism in normal subjects while awake and in the first period of non-REM sleep after placebo and zolpidem administration. Table 2 presents cortical values for absolute CGMR for whole brain and cortical areas. Whole cortex absolute metabolic rates were not statistically different on placebo and zolpidem nights by ANOVA (placebo versus zolpidem, $F=0.11$, $\mathrm{df}=1,9, p=.75$ ), even when the interaction with plasma level group was examined (drug condition by plasma level group, $F=$ $0.39, \mathrm{df}=1,9, p=.54$ ). There was a trend level effect only for the condition-by-lobe-by-segment-by-hemisphere interaction $(F=1.98$, Huynh-Feldt-corrected $\mathrm{df}=$ 9,81, $p=.0525$ ). The addition of plasma level group (above and below median) did not yield statistical significance for any interaction term with drug condition, and the simple interaction for the high plasma level group of drug condition by lobe by segment by hemisphere was also a trend $(F=1.95$, Huynh-Feldtadjusted $\mathrm{df}=9,81, p=.0567$; low plasma level group, $F=$ $0.86, p=.56, \mathrm{~ns})$.

The areas of the brain that showed significant correlations between plasma zolpidem levels and change in absolute values of glucose metabolic rate (zolpidem minus placebo nights) are shown in Figure 3. These results 
Table 1. Polygraphic Sleep Measures and EEG Baseline Crossings on Experimental FDG Infusion Nights with Placebo and Zolpidem (10 mg)

\begin{tabular}{lcr} 
Sleep Measures & Placebo & Zolpidem \\
\hline Goodnight time & $23: 11 \pm 1: 05$ & $23: 01 \pm 1: 11$ \\
Sleep Latency & $17 \pm 10$ & $16 \pm 8$ \\
Sleep onset to FDG infusion & $27 \pm 11$ & $22 \pm 9$ \\
Total time post-FDG infusion & $37 \pm 9$ & $32 \pm 2$ \\
Stage 1 sleep & $1 \pm 1$ & $1 \pm 1$ \\
Stage 2 sleep & $18-3$ & $11 \pm 8$ \\
Stage 3 sleep & $2 \pm 2$ & $2 \pm 2$ \\
Stage + sleep & $14=12$ & $18 \pm 9$ \\
Awake or movement time & $1=1$ & $1 \pm 1$ \\
& & \\
Baseline EEG crossings (Her $z)$ & & \\
following FDG or placebo & & \\
0.3 to $<1$ & $8.28-2.99$ & $10.13 \pm 4.35$ \\
1.0 to $<2$ & $30.87 \pm 7.60$ & $29.73 \pm 4.35$ \\
2.0 to $<3$ & $22.15 \pm 4.20$ & $20.55 \pm 4.37$ \\
3.0 to $<4$ & $15.35 \pm 3.75$ & $15.31 \pm 4.56$ \\
4.0 to $<8$ & $36.09 \pm 12.39$ & $31.94 \pm 9.23$ \\
8.0 to $<12$ & $25.26 \pm 8.50$ & $25.80 \pm 11.08$ \\
12.0 to 20 & $139.01 \pm 64.18$ & $161.12 \pm 156.63$
\end{tabular}

Sleep data are in minutes, except Goodnight time' (military time)

indicate that the higher the plasma concentration of zolpidem, the greater the reduction of absolute local cerebral glucose metabolic rate compared with placebo in selected areas of the brain. Among the 63 brain areas in each hemisphere that were explored (126 total correla- tions), 25 correlations were significant at the $p<.05$ two-tailed test level for absolute values, and all were negative. The negative correlations were concentrated in the medial frontal cortical and cingulate regions (five slice levels, anterior and posterior), frontal white mat-

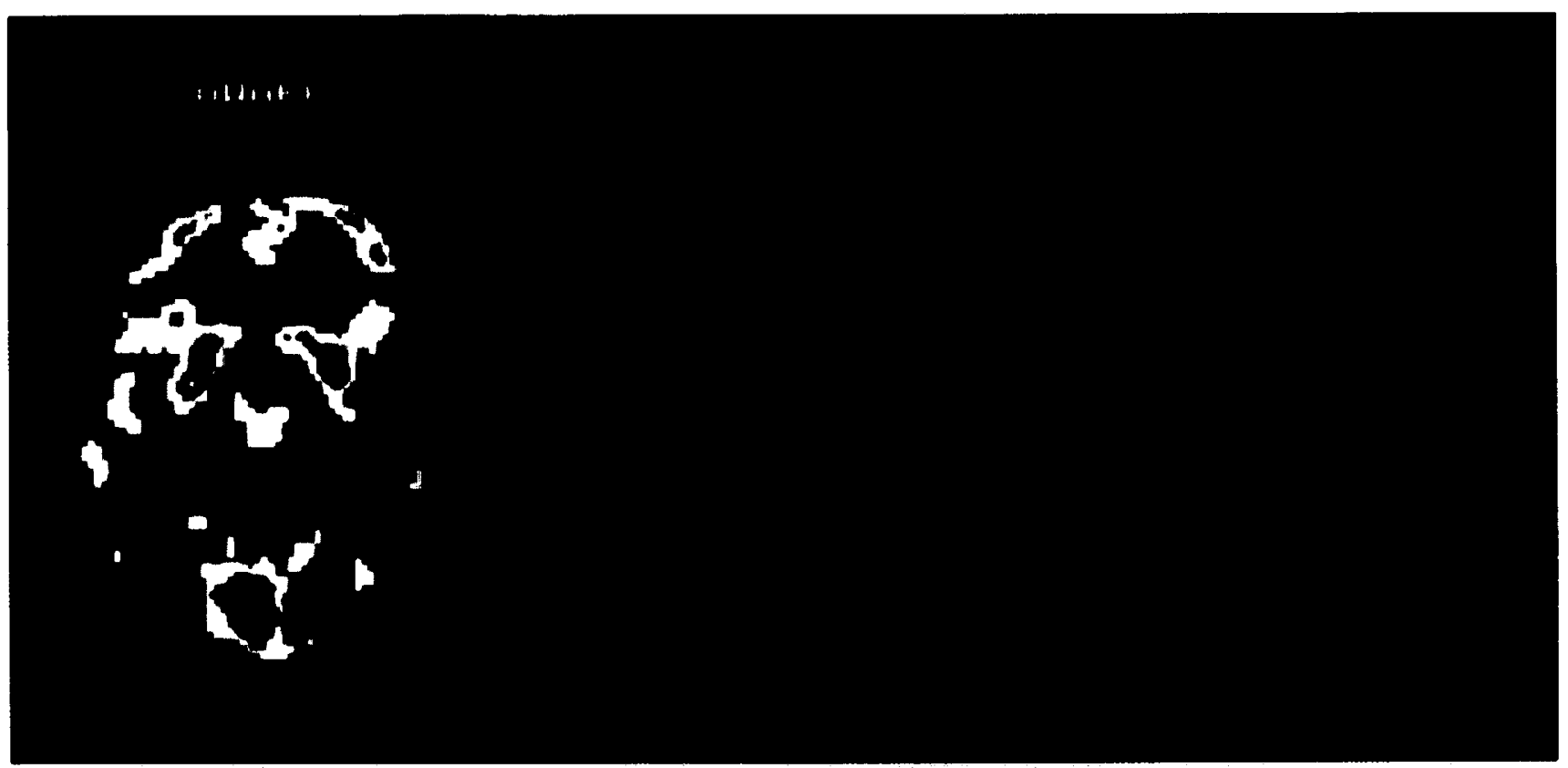

Figure 2. Comparison of absolute CGMRs in typical normal volunteers during wakefulness and the first non-REM period (following placebo and zolpidem, $10 \mathrm{mg}$ ). Scans are all shown on the same absolute metabolic scale from $51.0 \mathrm{micromoles}$ glucose $/ 100 \mathrm{~g} /$ minute (white) through red-violet, with violet beginning at 8.1 micromoles $/ 100 \mathrm{~g} / \mathrm{minute}$. The awake subject was not part of the present study but is shown to demonstrate that cerebral glucose metabolic rate during wakefulness is significantly higher than during non-REM sleep (Buchsbaum et al. 1989). The two scans taken during non-REM sleep suggest slightly lower localized cerebral glucose metabolic rate on the zolpidem night than on the placebo night. 
Table 2. CGMR (mmol $/ 100 \mathrm{~g} /$ minute) during the First Non-REM Period on Placebo and Zolpidem Nights

\begin{tabular}{lrr}
\hline Structure & Placebo & Zolpidem \\
\hline Whole brain & $19.2 \pm 3.4$ & $19.4 \pm 4.9$ \\
Frontal lobe & $20.5 \pm 3.1$ & $20.4 \pm 4.0$ \\
Parietal lobe & $20.2 \pm 2.8$ & $20.1 \pm 4.6$ \\
Temporal lobe & $16.7 \pm 3.5$ & $16.2 \pm 3.4$ \\
Occipital lobe & $19.6 \pm 3.1$ & $19.1 \pm 4.2$
\end{tabular}

ter, putamen, thalamus, and hippocampus. No lateral cortical area showed a significant correlation between change in metabolic rate and plasma concentration of zolpidem.

\section{Relative Metabolic Rate and Plasma Concentrations of Zolpidem}

Analyzing relative metabolic rate in the cortex similarly yielded only one trend-level interaction with plasma level group (median split) and drug condition (drug condition by plasma level class by lobe by hemisphere, $F=3.83, \mathrm{df}=3,8, p=.057$ ). Because we might expect an effect only in the high-plasma group, we examined simple interactions in the high-plasma level group only and found the drug condition by lobe by hemisphere interaction significant ( $F=4.86, \mathrm{df}=3,8, p=.03$ ). The relative metabolic rate tended to decrease more on the left than the right hemisphere and more in the lateral temporal and occipital than parietal and frontal regions in the subgroup with plasma zolpidem concentrations above the median (Table 3 ).

The correlations between plasma concentrations of zolpidem and changes in selected local relative cerebral glucose metabolic rates are shown in Table 4. The areas of the right hemisphere with significant n'satio' correlations (two-tailed test) included the paracentral lobule, medial frontal gyrus, frontal white matter, precuneus, posterior putamen, and midbrain (Table 3). Areas with significant megatice correlations in the left hemisphere included the posterior thalamus and anterior cingulate. Significant positive correlations were found in the right anterior thalamus, left frontal matter, and left superior colliculus.

\section{DISCUSSION}

These results suggest that zolpidem modifies absolute CGMR in some, but not all, neuroanatomical areas during non-REM sleep in normal volunteers in direct relationship to plasma concentrations of zolpidem. Zolpidem-induced changes in local absolute and relative cerebral glucose metabolism were not uniform, however, throughout the brain. The effects tend to be greatest in subcortical and midline cortical areas rather than lateral cortical areas. Zolpidem plasma concentrations were correlated with reduced absolute and relative metabolic rates in the cingulate. In addition, they were correlated with the relative metabolic rate in the cerebellum ( $\mu<.05$ by a one-tailed test). In animals, both areas have high densities of $B Z_{1}$ receptors. On the other hand, several other areas that are high in $\mathrm{BZ}_{1}$ receptors, such as the frontal-parietal and sensorimotor cortex, were apparently unaffected. In addition, glucose metabolism in some areas, such as the thalamus and putamen, that have relatively few $\mathrm{BZ}_{1}$ receptors, was also lower.

The relatively localized effects of zolpidem in this study raises questions of (1) where and how zolpidem acts in the brain; and (2) how well changes in local cerebral glucose metabolism, as measured by PET in humans, reflects both the mechanisms of action and the clinical effects of zolpidem.

The limitations of this study preclude definitive answers for these questions at this time. The variance of plasma concentrations of zolpidem in our study was greater and the peak was less than expected on the basis of earlier results. For example, in a previous study in 20 normal young volunteers, plasma concentrations following oral administration of zolpidem (10-mg capsule) reached their maximum value at $1.2 \pm 0.2$ hours (information on file, Investigational Drug Brochure, June, 1988, Lorex Pharmaceuticals, Skokie, IL). Peak values were $143 \pm 18 \mathrm{ng} / \mathrm{ml}$; bioavailability was $67.5 \pm 5.5 \%$. Similar findings have been reported after oral administration of zolpidem tablets, $10 \mathrm{mg}$ (Thénot et al. 1988). The plasma half-life was reported to be $1.7 \pm 0.2$ hours, respectively. In our study, plasma concentrations of zolpidem reached their maximum slightly earlier, about 60 minutes after the oral dose, and mean plasma concentrations remained constant for the next 30 minutes. Peak values (highest concentration for each subject), however, were $105 \pm 106 \mathrm{ng} / \mathrm{ml}$, about $75 \%$ of the value reported in the earlier reports. Plasma zolpidem concentrations in our subjects were approximately $56 \pm 47$ $\mathrm{ng} / \mathrm{ml}$ during the 30 minutes following the administration of FDG. The variability of zolpidem concentrations was impressive, with mean values during the FDG uptake ranging from near zero (three subjects) to $165 \mathrm{ng} /$ $\mathrm{ml}$. Because FDG was administered about 1 hour after the oral administration of zolpidem, the FDG uptake period overlapped with the maximal mean plasma (and 

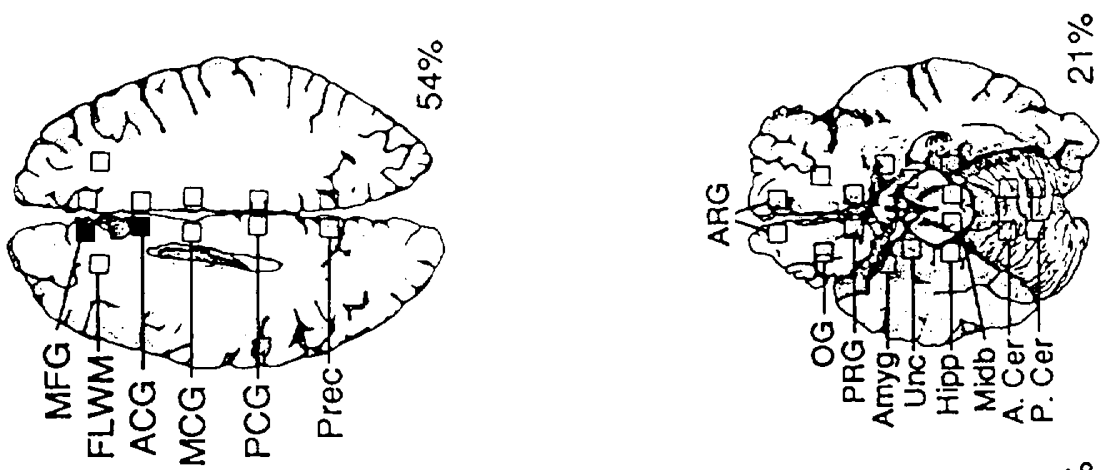

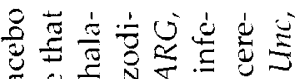
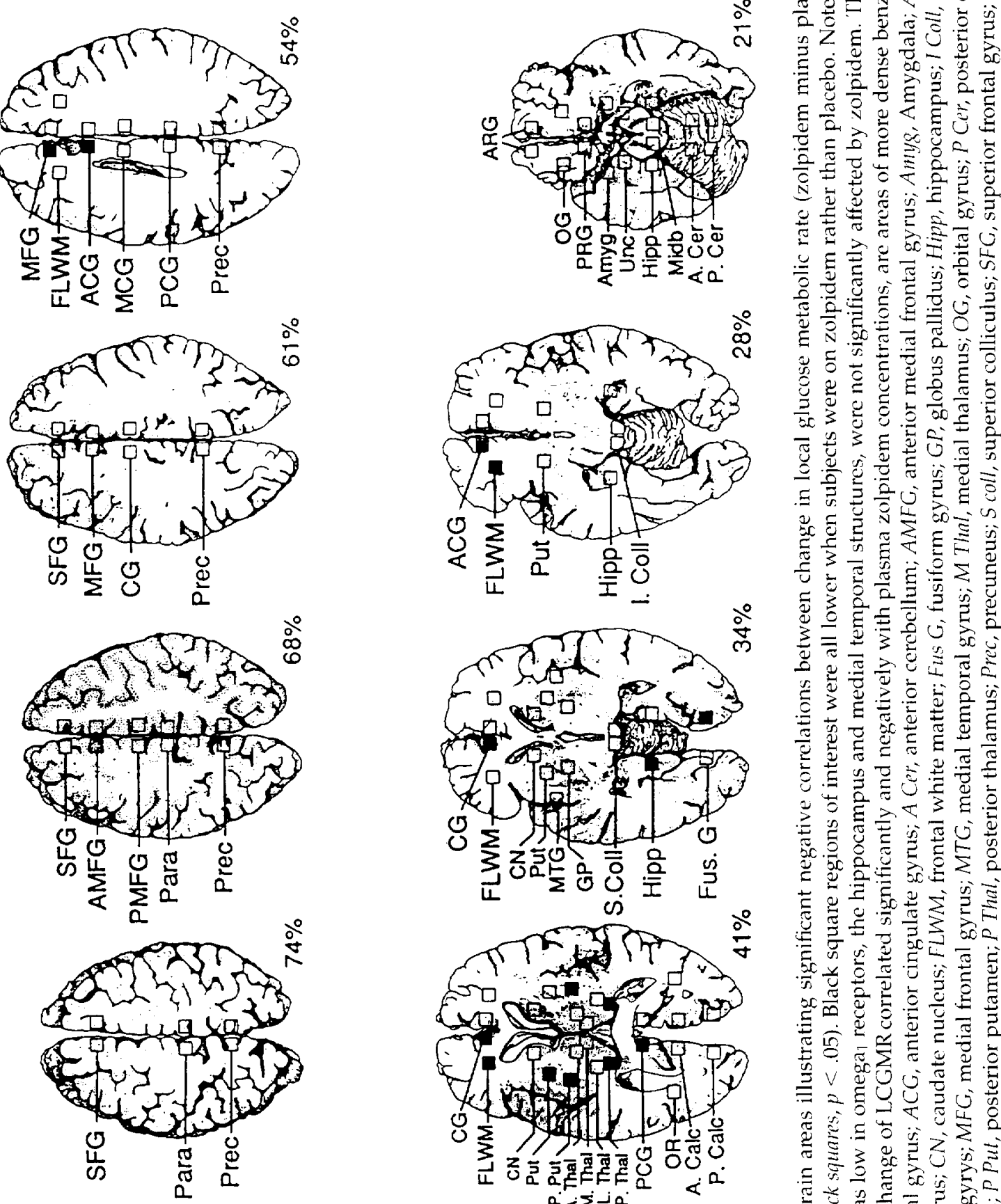

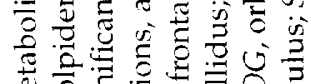

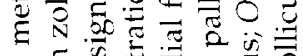
ऊิ

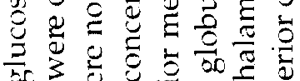

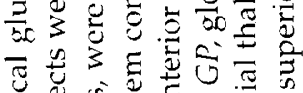

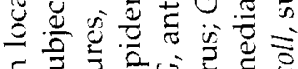

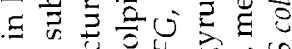

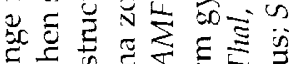

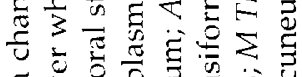

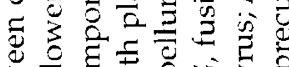

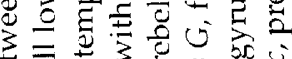

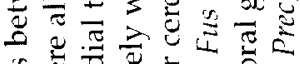

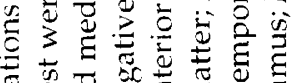

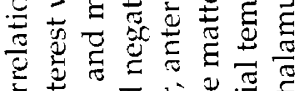

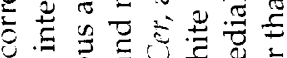

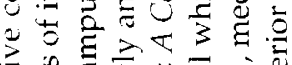

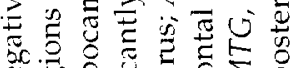

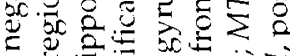

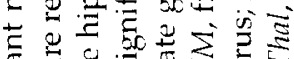
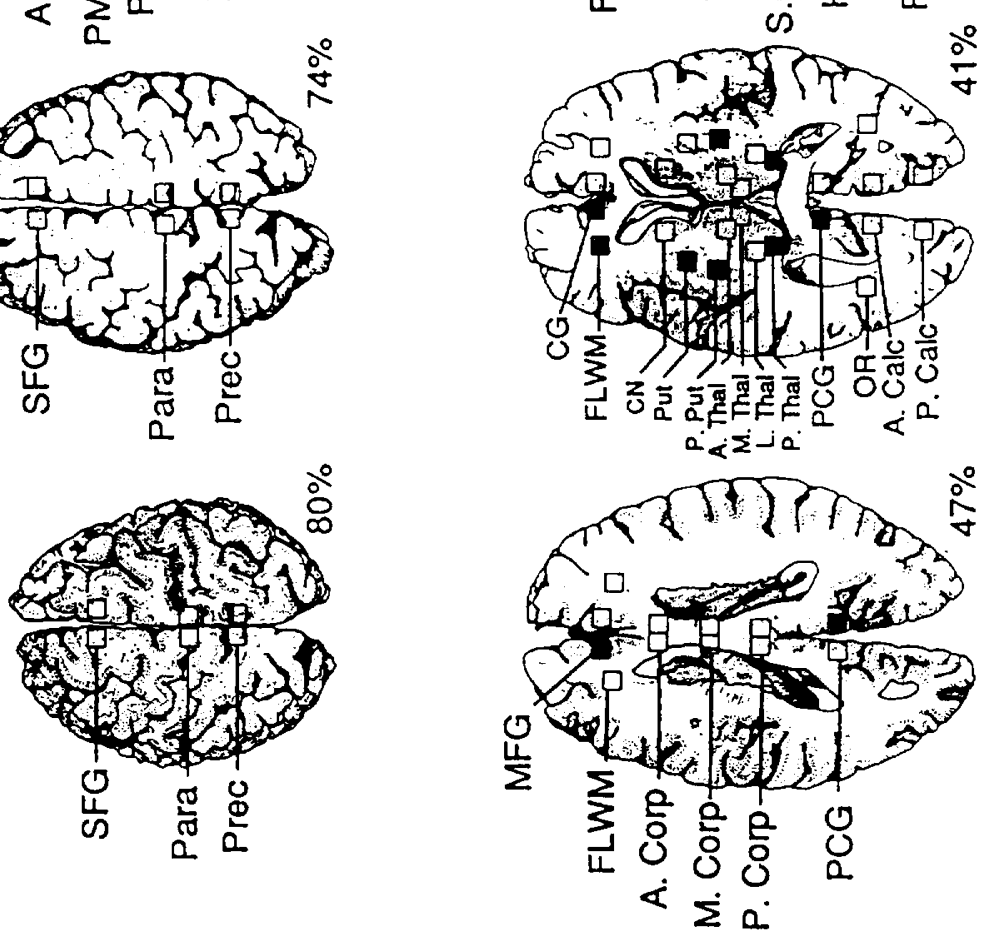

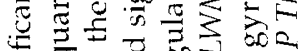

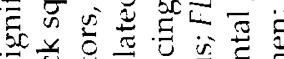
का

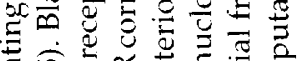

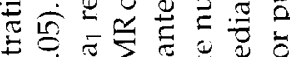

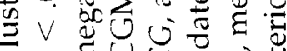
$\Rightarrow=$ ह

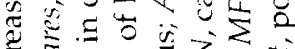

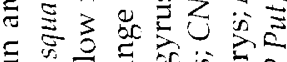

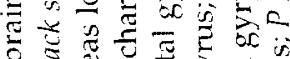

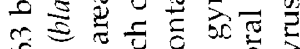

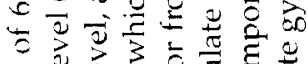

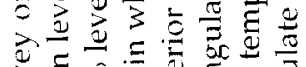

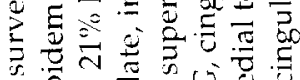

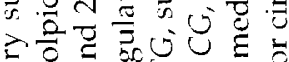

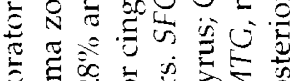

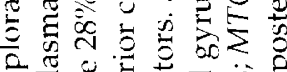

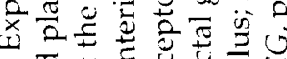
м

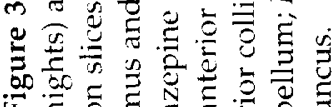


Table 3. Relative CGMR on Placebo and Zolpidem Nights

\begin{tabular}{lcccc}
\hline & \multicolumn{2}{c}{ Placebo } & \multicolumn{2}{c}{ Zolpidem } \\
& Left & Right & Left & Right \\
\hline Frontal & $1.14 \pm 0.07$ & $1.15 \pm 0.09$ & $1.14 \pm 0.07$ & $1.17 \pm 0.07$ \\
Parietal & $1.09 \pm 0.10$ & $1.17 \pm 0.09$ & $1.08 \pm 0.08$ & $1.16 \pm 0.08$ \\
Temporal & $0.91 \pm 0.11$ & $0.93 \pm 0.13$ & $0.89 \pm 0.13$ & $0.94 \pm 0.15$ \\
Occipital & $1.09 \pm 0.10$ & $1.10 \pm 0.10$ & $1.06 \pm 0.08$ & $1.08 \pm 0.09$ \\
\hline
\end{tabular}

MANOVA, treatment condition $\times$ hemisphere $\times$ lobe, $F=4.86, \mathrm{df}=3,8, p=.03$.

presumably brain) concentrations of zolpidem. In a study of rats, zolpidem was present in the brain at concentrations of about $30 \%$ to $50 \%$ of the plasma concentrations. Elimination from brain paralleled that in plasma (Garrigou-Gadenne et al. 1989). We have no explanation for the near-zero plasma zolpidem concentrations in three subjects, for the high variance in plasma pharmacokinetics, or for the generally low mean and peak plasma concentrations.

The unusually low concentration of zolpidem in our subjects limits the generalizability of our findings. Unlike some previous studies with zolpidem $(10 \mathrm{mg})$ in normal volunteers (Lund et al. 1988; Merlotti et al.
1989), we did not find reduced sleep latency or other significant effects on sleep parameters, including EEG crossings. Most of our cerebral metabolic findings emerged only after we correlated plasma concentrations of zolpidem with change in local cerebral metabolism. Even if we assume that the test-retest reliability for anatomical localization in FDG PET studies is high, the inherent anatomic resolution of PET may be insensitive to local metabolic activity. Insofar as our subjects had lower plasma concentrations of zolpidem than usually expected, our study may have underestimated the magnitude to which zolpidem typically affects brain glucose metabolism at customary plasma concentrations

Table 4. Medial Cortical and Subcortical Brain Regions: Change in Relative Values (zolpidem minus placebo nights) in Selected Regions of Interest as a Correlate of Plasma Concentrations of Zolpidem

\begin{tabular}{|c|c|c|c|}
\hline \multicolumn{2}{|c|}{ Slice Level ( $\%$ of brain height) } & \multirow{2}{*}{$\frac{\text { Left }}{0.223}$} & \multirow{2}{*}{$\frac{\text { Right }}{0.580^{*}}$} \\
\hline $74^{\prime \prime}$ & Paracentral lobule & & \\
\hline \multirow[t]{2}{*}{$68^{\circ} \circ$} & Post medial frontal gyrus & 0.467 & 0.365 \\
\hline & Precuneus & 0.241 & -0.466 \\
\hline \multirow[t]{2}{*}{$61^{\prime \prime} 0$} & Medial frontal gyrus & -0.503 & $0.549^{*}$ \\
\hline & Precuneus & 0.191 & $-0.544^{*}$ \\
\hline \multirow[t]{3}{*}{$54^{\prime \prime}$} & Frontal white matter & $0.606^{*}$ & 0.127 \\
\hline & Medial frontal gyrus & -0.400 & 0.308 \\
\hline & Middle cingulate & -0.401 & 0.442 \\
\hline \multirow[t]{2}{*}{$47^{\circ}{ }^{\circ}$} & Frontal white matter & 0.158 & 0.497 \\
\hline & Medial frontal gyrus & -0.495 & 0.466 \\
\hline \multirow[t]{6}{*}{$41^{\prime \prime} "$} & Frontal white matter & 0.442 & 0.043 \\
\hline & Posterior putamen & -0.000 & $-0.555^{*}$ \\
\hline & Anterior thalamus & 0.060 & $0.583 *$ \\
\hline & Medial thalamus & 0.041 & 0.234 \\
\hline & Lateral thalamus & -0.360 & -0.006 \\
\hline & Posterior thalamus & $-0.524^{*}$ & -0.414 \\
\hline \multirow[t]{4}{*}{$34^{\prime \prime} \circ$} & Anterior cingulate & $-0.537^{*}$ & -0.088 \\
\hline & Caudate nucleus & 0.471 & -0.222 \\
\hline & Gilobus palidus & 0.151 & -0.456 \\
\hline & Superior colliculus & $0.720^{*}$ & 0.264 \\
\hline $28^{\prime \prime \prime}$ & Frontal white matter & 0.280 & $-0.760^{*}$ \\
\hline \multirow[t]{3}{*}{$21^{\prime \prime}$} & Hippocampus & 0.401 & 0.376 \\
\hline & Midbrain & -0.472 & -0.584 \\
\hline & Anterior cerebellum & -0.489 & -0.503 \\
\hline
\end{tabular}

${ }^{*}<-0.532, f<.05$ for two-tailed test for hypothesis of larger metabolic decrease (zolpidem minus placebo) associated with higher plasma drug level on the active compound study day; $p>532$ for positive correlation with plasma zolpidem levels $(\mu<.05$, two-tailed test), $r=.458, p<.05$ for one-tailed test. For a theoretical Bonferroni adjustment, $r<-.823$ or $r>823, p<.0004$. 
during non-REM sleep.

Moreover, because of the large numbers of correlations performed between regions of interest and plasma concentrations of zolpidem, the possibility of false-positive findings is high and the results should be interpreted as exploratory. Nevertheless, the data indicate that local GMRC is sensitive to plasma drug levels.

Other investigators have used PET to study the effects of benzodiazepines on localized cerebral activity during wakefulness, for example, changes in LCGMR in the occipital and parietal areas following administration of clorazepate in patients with generalized anxiety disorder (Buchsbaum et al. 1987; Wu et al. 1991b). De Wit et al. (1991) also reported larger reductions of metabolic rate in cortex than thalamus following administration of benzodiazepines in normal waking controls, although in a small sample Foster et al. (1987) noted only a general metabolic decrease associated with benzodiazepine treatment. The distribution of benzodiazepine receptors in occipital cortex and subcortical structures was not altogether dissimilar in studies using radioactive ligands for the benzodiazepine receptor with PET (see review by Maziere and Maziere 1990). Using a beta-carboline suggested to preferentially bind to the $\mathrm{BZ}_{1}$ site, they observed that it displaced the labeled receptor ligand preferentially in the cerebellum, an area we also detected as regionally changed by zolpidem. It is noteworthy that the brain areas that showed the greatest change in metabolic rate are those areas found to have the highest density of benzodiazepine receptors in human autopsy studies (see Buchsbaum et al. 1987 for review).

Similar regional metabolic/receptor density relationships have been shown for the dopamine system in a number of studies of the effects of neuroleptic medication and glucose change in the basal ganglia (see Buchsbaum et al. 1992b). Because $\mathrm{BZ}_{1}$ receptors are distributed unevenly across the brain, we reported a survey of different neuroanatomical sites. Although a few areas are small, no more than one FWHM in each hemisphere, large $\left(30^{\circ},-50^{\circ} \circ\right)$ changes in metabolic rate might be detected at this sample size. Because no whole human brain survey exists of different types of $B Z$ receptor density, a simple regression line analysis of metabolic rate change against $B Z_{1}$ receptor density was not possible.

Even though some previous studies have demonstrated objective shortening of sleep latency with zolpidem (10 mg) in normal young volunteers (Lund et al. 1988; Merlotti et al. 1989), others, consistent with our own, did not observe shorter sleep latency (Nicholson and Pascoe 1986; Blois and Gaillard 1988; Brunner et al. 1991). Using spectral analysis of the EEG during nonREM sleep, Brunner et al. (1991) reported that zolpidem (10 mg), reduced power density in approximately the theta/alpha $(4-10 \mathrm{~Hz})$ frequency range and spindle/ beta $(14.25-15 \mathrm{~Hz})$ range during the first 2 hours of sleep in normal volunteers. To our knowledge, no previous investigators have reported the effects of zolpidem on the zero crossing EEG measures. For technical reasons, we were unable to perform a more comprehensive analysis of the EEG.

Our results have implications for the mechanisms by which hypnotics exert their sleep-promoting effects in patients with insomnia and in normal controls. Generally, treatment of insomnia involves three different strategies: (1) correction of circadian or biological rhythm abnormalities, as with adherence to an appropriate, regular sleep-wake cycle, therapeutic use of light-dark cycles or, possibly, melatonin; (2) reduction of arousal mechanisms, such as management of physical and psychic pain or distress or sleep in a safe and quiet environment; and (3) enhancement of sleep-promoting mechanisms at the appropriate bedtime, such as sleep hygiene and administration of sleeping pills.

Although some benzodiazepines may affect circadian rhythms in animals, it is not clear that this effect is clinically important in humans or that zolpidem affects circadian rhythms (Copinschi et al. 1995). Our study suggests that zolpidem may act through the two other interrelated factors, reducing physiological arousal mechanisms and enhancing physiological sleep-promoting mechanisms.

First, insofar as cerebral metabolism is directly related to arousal mechanisms, the sleep-inducing effects of zolpidem are apparently associated with reduced cerebral metabolism and arousal mechanisms. Horne and Shackell (1987), McGinty and Szymusiak (1990), and others have suggested that non-REM sleep and, in particular, delta sleep are enhanced when brain temperature falls. To our knowledge, no studies of cerebral metabolism have been conducted in nondepressed insomniac patients during sleep. Bonnet et al. $(1991,1992)$ have hypothesized, however, that whole-body hypermetabolic rates during sleep are elevated in primary insomniac patients. In a study of depressed patients, we report that overall cerebral glucose metabolic rate is significantly higher during the first non-REM period in depressed patients than in normal controls (Ho, Gillin, $\mathrm{Wu}$, Bunney, and Buchsbaum unpublished data). Other investigators have reported that core body temperature is elevated during sleep in depressed and insomnia patients (Avery et al. 1986; Monroe 1967). Because systemic glucose utilization is highly correlated with cerebral glucose metabolism during non-REM sleep (Boyle et al. 1994), it is possible that higher cerebral glucose metabolism increases core body temperature and wholebody metabolism during sleep more in insomniac patients compared than in normal controls. A testable set of hypotheses for future studies includes: (1) Insomniac patients have higher core body metabolism, higher whole-body metabolic rates, and increased whole-brain CGMR during non-REM sleep (and possible REM 
sleep) than normal controls; (2) Zolpidem or other hypnotic agents correct abnormally high rates of wholebrain, localized CGMR, or whole-body metabolism in patients with insomnia; (3) because hypnotic-induced improvement in sleep in insomniac patients appears to vary directly with the severity of sleep disturbance prior to treatment (Gillin et al. 1980), the hypnotic-induced reduction of metabolism may vary with the severity of objective insomnia.

Second, zolpidem may directly promote sleep-induced physiological mechanisms in the brain. Many neuroanatomical areas are implicated in the regulation of nonREM sleep, including the cerebral cortex, basal forebrain, anterior hypothalamus, thalamus, and tractus solitarias, whereas projections from the posterior hypothalamus, dorsal tegmentum, the reticular activating system, and, possibly, the suprachiasmatic nucleus maintain cortical arousal and EEG desynchronization during wakefulness and REM sleep. Nevertheless, no neuroanatomical center has been shown to be uniquely necessary for non-REM sleep, REM sleep, or wakefulness. Insofar as zolpidem affects $B Z_{1}$ receptors, it may be enhancing or mimicking the effects of GABA, which are known to be involved in sleep-promoting processes in the thalamus and elsewhere.

The observation that local CGMR in the hippocampus, an area involved in memory, was lower is interesting in light of reports indicating that benzodiazepine and imidazopyridine hypnotics may induce amnesia. In summary, zolpidem appears to decrease local absolute CGMR in selected brain areas during non-REM sleep in normal volunteers proportionately to plasma zolpidem concentrations. The reduction of local CGMR was not uniform across the brain and was modestly related to the known regional distribution of $B Z_{1}$ receptors. Further studies are needed to determine the effect of zolpidem and other hypnotics on cerebral metabolism during sleep in normal controls and in patients with sleep disorders.

\section{ACKNOWLEDGMENTS}

These studies were supported in part by the Lorex Corporation and G.D. Searle Company, the University of California Brain Imaging Committee, the UCSD Mental Health Clinical Research Center (MH30914), the UCSD Fellowship in Clinical Psychobiology and Psychopharmacology for CH (MH18399), a Merit Review grant from the Veterans Administration, grants from the National Institute of Mental Health to JCG (MH38738), MSB (MH41282), and JCW (MH445165).

\section{REFERENCES}

Avery DH, Wildschiodtz G, Smallwood RG, Martin D, Rafaelson OJ (1986): REM latency and core temperature relationships in primary depression. Acta Psychiatr Scand 74:269-280

Bartlett EJ, Barouche F, Brodie JD, Wolkin A, Angrist B, Rotrosen J, Wolf AP (1991): Stability of resting deoxyglucose metabolic values in PET studies in schizophrenia. Psychiatry Res Neuroimag 40:11-22

Benavides J, Peny B, Durand A, Arbilla S, Scatton B (1993a): Comparative in vivo and in vitro regional selectivity of central omega (benzodiazepine) site ligands in inhibiting $[3 \mathrm{H}]$ flumazenil binding in the rat central nervous system. J Pharmacol Exp Ther 263:884-896

Benavides J, Peny B, Ruano D, Victorica J, Scatton B (1993b): Comparative autoradiographic distribution of central omega (benzodiazepine) modulatory site subtypes with high, intermediate and low affinity for zolpidem and alpidem. Brain Res 604:240-250

Blois R, Gaillard JM (1988): The effects of zolpidem on characteristics of normal human sleep. In Sauvanet JP, Langer SZ, Morselli PL (eds), Imidazopyridines in Sleep Disorders. New York, Raven, pp. 375-376

Bonnet MH, Arand DL (1992): Caffeine use as a model of acute and chronic insomnia. Sleep 15:526-536

Bonnet MH, Berry RB, Arand DL (1991): Metabolism during normal, fragmented, and recovery sleep. J Appl Physiol 71:1112-1118

Boyle PJ, Scott JC, Krentz AJ, Nagy RJ, Comstack E, Hoffman $C$ (1994): Diminished brain glucose metabolism is a sufficient determinant for falling rates of systemic glucose utilization during sleep in normal humans. J Clin Invest 93:529-535

Brunner DP, Dijk D-J, Münch M, Borbély AA (1991): Effect of zolpidem on sleep and sleep EEG spectra in healthy young men. Psychopharmacology 104:1-5

Buchsbaum MS, Delisi LE, Holcomb H, Capelletti J, King AC, Johnson J, Hazlett E, Post RM, Morihisa J, Carpenter W, Cohen R, Pickar D, Kessler R (1984): Anteriorposterior gradients in cerebral glucose metabolism use in schizophrenia and affective disorders. Arch Gen Psychiatry 41:1159-1166

Buchsbaum MS, Wu JC, Haier RJ, Hazlett E, Ball R, Katz M, Sokolski K, Lagunas-Solar M, Langer DH (1987): Positron emission tomography assessment of effects of benzodiazepines on regional glucose metabolic rate in patients with anxiety disorder. Life Sci 40:2393-2400

Buchsbaum MS, Gillin JC, Wu J, Hazlett E, Sicotte N, Dupont RM (1989): Regional cerebral glucose metabolic rate in human sleep assessed by positron emission tomography. Life Sci 45:1349-1356

Buchsbaum MS, Potkin S, Marshall J, Lottenberg S, Teng CY, Heh CW, Tafalla R, Reynolds C, Abel L, Plon L, Bunney WE (1992a): Effects of clozapine and thiothixene on glucose metabolic rate in schizophrenia. Neuropsychopharmacology 6:155-163

Buchsbaum MS, Potkin SG, Siegel BV, Lohr J, Katz M, Gottschalk LA, Marshall JF, Lottenberg S, Tang CY, Abel L, PIon L, Bunney WE (1992b): Striatal Metabolic Rate and Clinical Response to Neuroleptics in Schizophrenia. Arch Gen Psychiatry 49:966-974

Copinschi G, Akseli E, Moreno-Reyes R, Leproult R, L'Hermite-Balériauz $M$, Caufriez $A$, Vertongen $F$, and Van Cauter E (1995): Effects of bedtime administration of 
zolpidem on circadian and sleep-related hormonal profiles in normal women. Sleep 18:417-424

de Wit H, Metz J, Wagner N, Cooper M (1991): Effects of diazepam on cerebral metabolism and mood in normal volunteers. Neuropsychopharmacology 5:33-41

Fairweather DB, Kerr JS, Hindmarch I (1992): The effects of acute and repeated doses of zolpidem on subjective sleep, psychomotor performance and cognitive function in elderly volunteers. Eur I Clin Pharmacol 43:597-601

Foster NL, Van Der Spek AF, Aldrich MS, Berent S, Hichwa RH, Sackellares JC, Gilman S, Agranoff BW (1987): The effect of diazepam sedation on cerebral glucose metabolism in Alzheimer's disease as measured using positron emission tomography. J Cereb Blood Flow Metab 7:415420

Franzini C (1992): Brain metabolism and blood flow during sleep. I Sleep Res 1:3-16

Garrigou-Gadenne D, Burke JT, Durand A, Depoortere H, Thénot JP, Morselli I'L (1989): Pharmacokinetics, brain distribution and pharmaco-electrocorticographic profile of zolpidem, a new hypnotic, in the rat. I Pharmacol Exp Ther 248:1283-1288

Gillin JC, Mendelson WB (1980): Sleeping pills. For whom? When? How Long? In Palmer GC (ed), Neuropharmacology of Central Nervous Svstem and Behavioral Disorders. New York, Academic Press, pp. 285-316

Guinebault P, et al. (1986): High performance liquid chromatography determination of zolpidem, a new sleep inducer, in biolouical fluids with fluorometric detection. J Chromatogr 383:206-211

Harris GJ, Links JM, Pearlson GD, Camargo EE (1991): Cortical circumferential profile of SPECT cerebral perfusion in Alzheimer's disease. Psych Res Neuroimag 40:167-180

Horne JA, Shackell BS (1987): Slow wave sleep after body heating: Proximity to sleep and effects of aspirin. Sleep $10: 383-392$

Jonas JM, Coleman BS, Sheridan AQ, Kalinske RW (1992): Comparative clinical profiles of triazolam versus other shorter-acting hypnotics. J Clin Psychiatry 53(suppl):1933

Kennedy C, Gillin JC, Mendelson WB, et al. (1982): Local cerebral glucose utilization in Non-REM sleep. Nature 297:325-327

Langer SZ, Arbilla S (1988): Imidazopyridines as a tool for the characterization of benzodiazepine receptors: A proposal for a pharmacological classification as omega receptor subtypes. Pharmacol Biochem Behav 29(4):763766

Lund R, Rüther E, Wober W, Hippius H (1988): Effects of zolpidem (10) and $20 \mathrm{mg}$ ), lorazepam, triazolam and placebo on night sleep and residual effects during the day In Sauranet JP, Langer SZ, Morselli PL (eds), Imidazopyridines in Sleep Disorders. New York, Raven, pp $193-204$

Maarek L, Cramer I, Attali P, Coquelin IP, Morselli PL (1992) The safety and efficacy of zolpidem in insomniac patients: A long-term open study in general practice. I Int Med Res 20(2):162-170

McGinty D, Szymusiak R (1990): Keeping cool: A hypothesis about the mechanisms and functions of slow wave sleep. Trends Neurosci 13:480-487
Madsen P, Vorstrup S (1991a): Cerebral blood flow and metabolism during sleep. Cerebrovasc Brain Metab Rev 3:281-296

Madsen P, Schmidt JF, Wildschiodtz G, et al. (1991b): Cerebral $\mathrm{O}_{2}$ metabolism and cerebral blood flow in humans during sleep and rapid-eye-movement sleep. J Appl Physiol 80:2597-2601

Marksitzer R, Benke D, Fritschy JM, Trzeciak A, Bannwarth

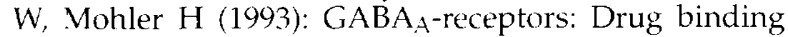
profile and distribution of receptors containing the alpha ${ }_{2}$-subunit in situ. J Recept Res 13(1-4):467-477

Maquet P, Dive D, Salmon E, et al. (1992): Cerebral glucose utilization during stage 2 sleep in man. Brain Res 571:149-153

Maziere B, Maziere M (1990): Where have we gone to with neuroreceptor mapping of the human brain? Eur J Nuc Med 16:817-835

Merlotti L, Roehrs T, Koshorek G, Zorick F, Lamphere J, Roth $T$ (1989): The dose effects of zolpidem on the sleep of healthy normals. J Clin Pharmacol 1:9-14

Mertens S, Benke D, Mohler H (1993): GABA receptor populations with novel subunit combinations and drugs binding profiles identified in brain by alpha5- and deltasubunit-specific immunopurification. J Biol Chem 268 (8):5965-5973

Monroe LJ (1967): Psychological and physiological differences between good and bad sleepers. J Abnorm Psychol 72:255-264

Nakamura R, Kennedy C, Gillin JC, et al. (1983): Hypnogenic center theory of sleep: No support from metabolic mapping in monkeys. Brain Res 268:372-376

Nicholson AN, Pascoe PA (1986): Hypnotic activity of an imidazolpyridine (zolpidem). Brit ] Clin Pharmacol 21:205211

Piercey MF, Hoffman WE, Cooper M (1991): The hypnotics triazolam and zolpidem have identical metabolic effects throughout the brain: Implications for benzodiazepine receptor subtypes. Brain Res 554(1-2):244-252

Ramm P, Frost BJ (1983): Regional activity in rat brain during sleep wake activity. Sleep 6:196-216

Ramm P, Frost BJ (1986): Cerebral and local glucose cerebral metabolism in the cat during slow wave and REM sleep. Brain Res 365:112-124

Rechtschaffen A, Kales A (1971): A manual of standard techniques and scoring system for sleep stages in human subjects. Washington, DC, Superintendent of Documents, U.S. Government Printing Office

Roger M, Attali P, Coguelin JP (1993): Multicenter, doubleblind, controlled comparison of zolpidem and triazolam in elderly patients with insomnia. Clin Ther 15(1):127136

Ruano, D, Benavides J, Machado A, Victorica J (1993): Regional differences in the enhancement of CABA of $[3 \mathrm{H}]$ zolpidem binding to omega 1 sites in rat brain membranes and sections. Brain Res 600(1):134-140

Scharf MB (1991): Dose response effects of zolpidem in normal geriatric subjects. J Clin Psychiatry 52:77-83

Shaw SH, Curson H, Coquelin IP (1992): A double-blind, comparative study of zolpidem and placebo in the treatment of insomnia in elderly psychiatric patients. Neuropsychopharmacology $7(1): 1-5$ 
Thénot JP, Hermann P, Durand A, Burke JJ, Allen J, Garrigou D, Vajta S, Albin H, Thébault JJ, Olive E, Warrington SJ (1988): Pharmacokinetics and metabolism of zolpidem in various animal species and in humans. In Sauvanet JP, Langer SZ, Morselli PL (eds), Imidazopyridines in Sleep Disorders. New York, Raven, pp 139-153

Wafford KA, Whiting PI, Kemp JA (1993): Differences in affinity and efficacy of benzodiazepine receptor ligands are recombinant gamma-aminobutyric acid A receptor subtypes. Mol Pharmacol 43(2):240-244

Wu JC, Gillin JC, Buchsbaum MS, Hazlett E, Sicotte N, Bun- ney WE Jr (1991a): The effect of sleep deprivation on cerebral glucose metabolic rate in normal humans assessed with positron emission tomography. Sleep 14:155-162

Wu JC, Buchsbaum MS, Hershey TG, Hazlett E, Sicotte N (1991b): PET in generalized anxiety disorder. Biol Psychiatry 29:1181-1199

Wu JC, Gillin JC, Buchsbaum MS, Hershey T, Johnson IC, Bunney WE (1992): Effect of sleep deprivation on brain metabolism of depressed patients. Am J Psychiatry 149: $538-543$ 